\title{
Pulsed-Power System for Leachate Treatment Applications
}

\author{
Sung-Roc Jang ${ }^{\dagger}$, Hong-Je Ryoo*, and Seung-Bok Ok** \\ $\dagger^{* *}$ Department of Energy Conversion Technology, University of Science \& Technology, Daejeon, Korea \\ ${ }^{*}$ Electric Propulsion Research Center, Korea Electrotechnology Research Institute, Changwon, Korea
}

\begin{abstract}
This paper presents a water treatment system for leachate from sewage-filled ground that uses a pulsed-power modulator developed based on semiconductor switches in order to realize a long life, a high repetition rate, and a fast rising time. The specifications of the developed pulsed-power modulator are the pulsed output voltage, the output current, the pulse repetition rate (PRR), the pulse width, and an average output power of $60 \mathrm{kV}_{\max }, 300 \mathrm{~A}_{\max }, 3000,50 \mu \mathrm{s}$, and $15 \mathrm{~kW}$, respectively. The pulsed-power water treatment system was introduced and analyzed using an equivalent electrical circuit model to optimize the output voltage waveform. The experimental results verify that the proposed water treatment system can be effectively used for industrial applications.
\end{abstract}

Key Words: Solid-state pulsed-power modulator, Water treatment system

\section{INTRODUCTION}

Leachate from sewage-filled ground is generally treated with chemical or biological methods to conform to environmental regulations pertaining to chemical oxygen demand (COD). However, such methods have a few disadvantages such as high cost, the requirement of large facilities, the generation of chemical reagents, and a long treatment time. Moreover, many chemical compounds such as those of the benzene series are difficult to decompose using these methods. In order to overcome these disadvantages, various studies have recently focused on pulsed-power water treatment systems. [1]-[4] Dielectric barrier discharge (DBD) in a water-air medium, a non-thermal plasma, generates many radicals such as ozone $\left(\mathrm{O}_{3}\right)$, atomic oxygen $(\mathrm{O})$, and hydroxyl ions $\left(\mathrm{OH}^{-}\right)$that can oxidize a wide range of impurities in water containing bacteria. Although pulsed-power treatment systems are superior to chemical and biological methods from the viewpoint of cost and efficiency, they are typically not used industrially because of the difficulties involved in producing pulsed power.

Designing pulsed-power modulators for water treatment involves many considerations such as the proper output voltage with respect to the dimensions of the plasma reactor, the fast rising time of the output pulse, the high repetition rate, and a long maintenance life. In order to generate high-voltage pulses,

\footnotetext{
Manuscript received Sep. 6, 2010; revised May 6, 2011

Recommended for publication by Associate Editor Tae-Woong Kim.

$\dagger$ Corresponding Author: scion10@keri.re.kr

Tel: +82-55-280-1426, Fax: +82-55-280-1490, Univ. of Science \& Tech.

* Electric Propulsion Research Center, Korea Electrotechnology Research Institute, Korea

** Department of Energy Conversion Technology, University of Science \& Technology, Korea
}

gas switches such as spark gaps or thyratrons are usually used owing to their high voltage and current ratings and their ease of drive. However, it is very difficult and occasionally impossible to generate a rectangular pulse with a controllable output voltage and pulse width. In addition, the generator has a limited life because the mechanical electrode eventually wears out. Therefore, solid-state pulsed-power modulators, made using a semiconductor switch instead of a gas switch, have been focused on. [5]-[9]

This paper describes a water treatment system with a novel solid-state pulsed-power modulator that takes into account the abovementioned considerations.

\section{Solid-State Pulsed-Power Modulator}

\section{A. Solid-State Pulsed-Power Modulator for Leachate Treat-} ment

The specifications of the developed pulsed-power modulator are summarized in Table I. The basic structure of the developed pulsed-power modulator is similar to the Marx generator, where the capacitor is charged separately and all of the capacitors are connected in series to generate a high voltage through the semiconductor switches. [9] The overall structure of the developed pulsed-power modulator is shown in Fig. 1. It can be divided into three main parts: (1) the charging inverter contained in each power cell that supplies the charging current to the storage capacitor through the charging loop and full-wave rectifier. (2) the power module (Module1-9) constructed using eight power cells that include an insulatedgate bipolar transistor (IGBT), a storage capacitor, and diodes (rectifier and bypass diodes). In order to generate the $60-\mathrm{kV}$ output pulse, nine power modules were connected in series. 
TABLE I

SPECIFICATIONS OF DEVELOPED SOLID-STATE PULSED-POWER MODULATOR

\begin{tabular}{|c|c|}
\hline Input voltage & 3-Phase $380 \mathrm{Vac}$ \\
Pulse output voltage & $0-60 \mathrm{kV}$ \\
Pulse output current & $0-300 \mathrm{~A}$ \\
Pulse width & $2-50 \mu \mathrm{s}$ \\
Pulse repetition rate & $20-3000 \mathrm{pps}$ \\
Pulse rising time & Less than $500 \mathrm{~ns}$ \\
Average output power & $15 \mathrm{~kW}$ \\
Charger efficiency & Greater than $90 \%$ \\
\hline
\end{tabular}

(3) the gate drive circuit where a full-bridge inverter serves as the last part and drives the 72 IGBTs simultaneously. The full-bridge inverter supplies the power and signals for all of the IGBTs through the control loop.

\section{B. Design of the Capacitor Charging Inverter}

In order to charge the 72 storage capacitors $(1000 \mathrm{~V}, 120$ $\mathrm{uF}$ ), series resonant inverter discontinuous conduction mode (DCM) operation was used because of its current source characteristics. The output current of a series resonant converter in DCM operation does not depend on the load conditions but on the ratio of the resonant frequency to the switching frequency. That is, when both frequencies are constant, the charging current is also constant irrespective of the storage capacitor voltage and load resistance. In addition, the four switches $\left(\mathrm{S}_{1}-\mathrm{S}_{4}\right)$, shown in Fig. 1, are turned on under zero current and turned off under zero current and zero voltage owing to the discontinuous resonant current and the anti-parallel diode conduction. This results in a high efficiency with reduced switching losses and a high switching frequency, which in turn reduces the size of the reactive components. Moreover, because the resonant inductor $\left(\mathrm{L}_{\mathrm{r}}\right)$ can be implemented by the leakage inductance of the high-voltage transformer without an additional inductor, the power density is very high. The detailed operational principle of this topology was first introduced with an average power of $10 \mathrm{~kW}$. [9] However, for high-power applications such as water treatment systems, which require a high repetition rate with a high output voltage, an average output power of $10 \mathrm{~kW}$ is insufficient. In this paper, to increase the average output power from 10 to $15 \mathrm{~kW}$, the charger was modified while maintaining the same topology.

As described above, the output current can be increased by increasing the switching frequency at a fixed resonant frequency. However, this generates high losses at the IGBTs and in the primary winding of the high-voltage transformer.

The main constraint for increasing the switching frequency is the conduction loss that occurs at the primary winding of the one turn transformer owing to the relatively high rms value of the resonant current. In addition, the high-frequency current through the high-voltage cable decreases the effective crosssectional area of the conductor owing to the decreased skin depth, which is inversely proportional to the square root of the switching frequency. In order to overcome these problems, two approaches have been proposed. The first is decreasing the resonant frequency by increasing the resonant tank parameters $\left(\mathrm{L}_{\mathrm{r}}, \mathrm{C}_{\mathrm{r}}\right)$. Increasing the resonant inductance is preferred because it offers two advantages. A higher value for the resonant inductance results in a lower value for the resonant

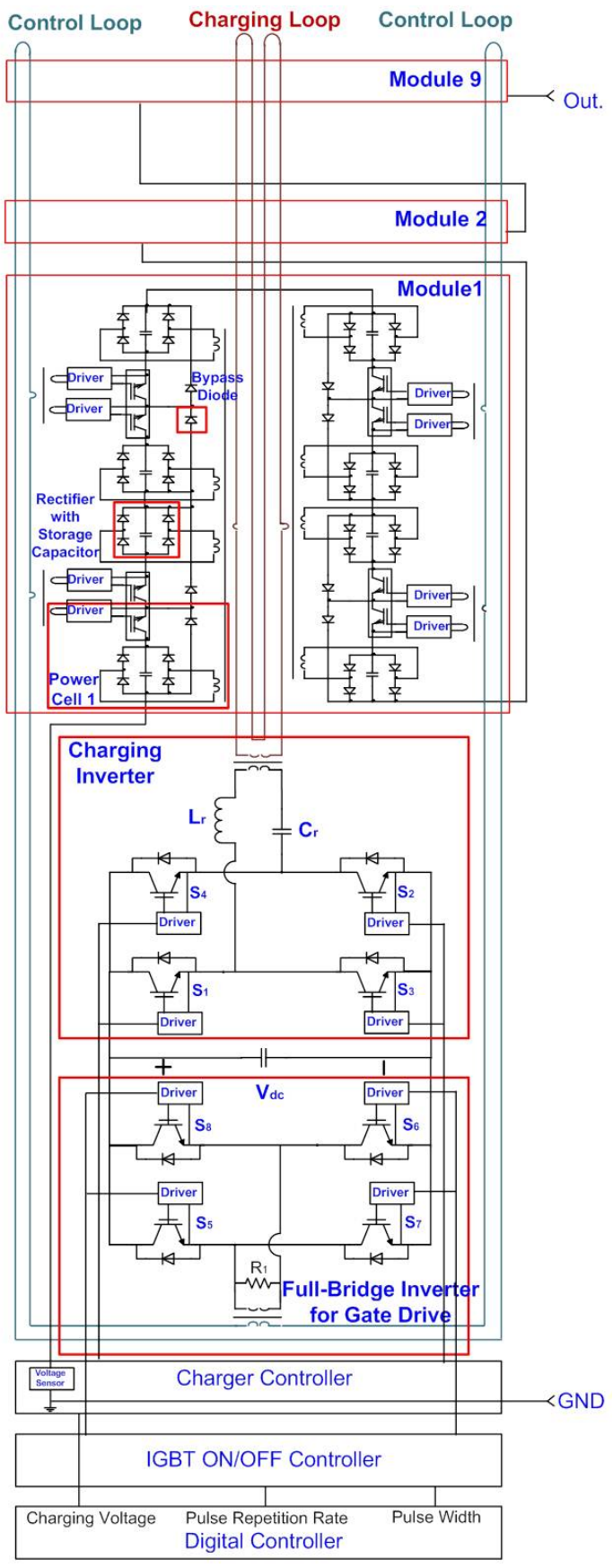

Fig. 1. Overall structure of solid-state pulsed-power modulator.

peak current by increasing the characteristic impedance of the resonant tank, and then, the rms value decreases. Moreover, it can be increased by separating the primary and secondary windings to an extent without any additional components being required. It is evident that a decreased resonant frequency for the same switching frequency increases the value of the output current, and its increased value is proportional to the ratio of both frequencies. Based on the modified design, the changed parameters are summarized in Table II. The frequency ratio in Table II clearly indicates that the output current increased 1.5 times for the same output voltage.

Although this method is helpful for decreasing the conduction losses for the same output power, it is not sufficient to increase the average output power by up to $50 \%$. Finally, a 
TABLE II

MODIFIED PARAMETERS

\begin{tabular}{|c|c|c|}
\hline Parameters & For $10 \mathrm{~kW}$ & For $15 \mathrm{~kW}$ \\
Resonant capacitance & $0.375 \mathrm{uF}$ & $0.46 \mathrm{uF}$ \\
Resonant inductance & $5.2 \mu \mathrm{H}$ & $8 \mu \mathrm{H}$ \\
Resonant frequency & $114 \mathrm{kHz}$ & $83 \mathrm{kHz}$ \\
Switching frequency & $30 \mathrm{kHz}$ & $33 \mathrm{kHz}$ \\
Frequency ratio & 0.263 & 0.398 \\
\hline
\end{tabular}

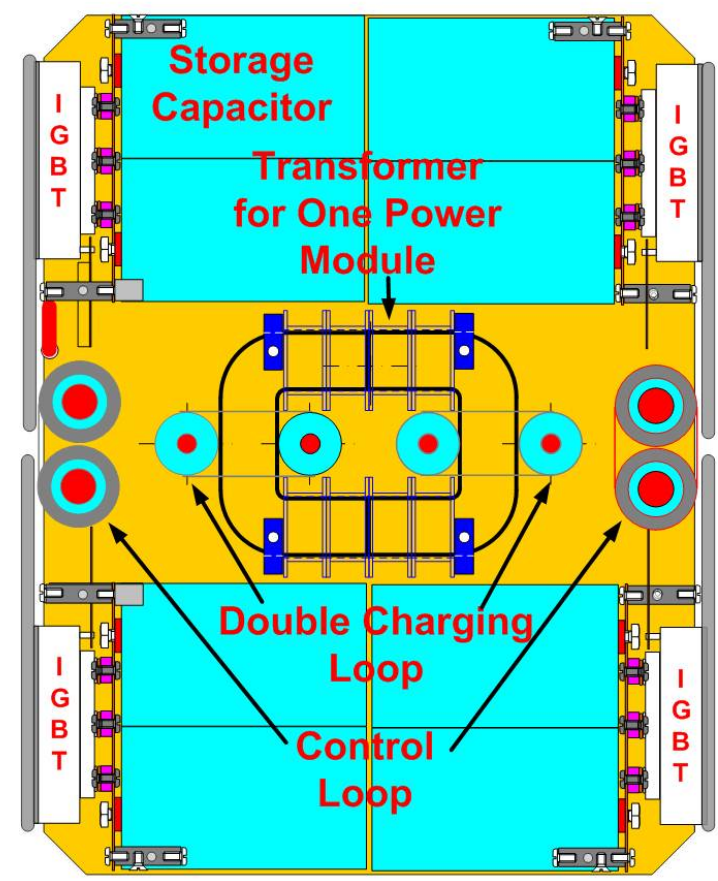

Fig. 2. Structure of high-voltage transformer.

configuration having two turns of primary winding in parallel was adopted. The modified structure of the high-voltage transformer with two turns of primary winding is shown in Fig. 2. To increase the leakage inductance of the transformer, a double charging loop, which represents the primary winding, was wound at the side of the secondary windings. In order to verify the modified design, a PSpice simulation model was developed, as shown in Fig. 3. The load of the capacitor charger was made equal to the resistance for $15 \mathrm{~kW}, 60 \mathrm{kV}$.

The simulation results shown in Fig. 4 validate the modified design in that the desired output power $(15 \mathrm{~kW})$, the voltage $(60 \mathrm{kV})$, and the resonant current for maximum power operation with a switching signal are obtained.

\section{Structure of Power Modules}

The structure of the power module is shown in Fig. 1. One power module consists of eight power cells that are connected to the secondary windings of the double charging loop. The storage capacitor in each power cell is charged to up to 850 $\mathrm{V}$ by the double charging loop through the full-wave rectifier.

When all of the IGBTs are simultaneously turned on, eight storage capacitors get connected in series, and then, the power module can generate eight times the voltage of one capacitor. That is, one power module can generate a voltage of up to $6.8 \mathrm{kV}$. In order to generate $60 \mathrm{kV}$, nine power modules connected in series through IGBTs with the same structure are required. The main advantage of this structure is that each IGBT has to withstand only the voltage of one capacitor even when there are IGBT on/off synchronization problems. Bypass diodes protect the IGBT from voltage surges. Fig. 5 shows the structure and the connection of two power modules.

\section{Gate Drive Circuit with a Full-Bridge Inverter}

The full-bridge inverter of a digital controller, shown in Fig. 1, receives the switching signal to generate on and off pulses based on the reference frequency and the pulse width command. Moreover, the full-bridge inverter generates and transfers both the power and the signal through the control loop to the proposed gate driver circuit, shown in Fig. 6. It does not require an additional isolated power for feeding the gate circuit or an optic cable for signal transfer. Furthermore, it exhibits very good short circuit protection characteristics. A high-voltage cable with adequate isolation, which is designed as a control loop, passes through the toroidal core of control transformer T1 forming a primary winding in a single turn. The direction and the real level of the current in the control loop are very important design factors in the proposed scheme. Because all of the signals and the power required to operate all of the gates of the IGBTs in series are delivered by the control loop current using a single-turn high-voltage cable, there is a significant reduction in the system's complexity and cost.

Because the power switch is controlled via a short pulse, with currents in different directions that mark the body of the real control pulse, it can easily adjust the pulse width during operation. Based on the operation modes, shown in Fig. 7, the operational principle can be analyzed as follows:

1) Pre-charging mode: Every working cycle begins with several turn-off pulses in order to pre-charge the storage capacitor $\mathrm{C} 1$. The operational principle is shown in Fig. 7. In order to obtain sufficient gate drive power, a charging routine can be performed, in which a turn-on and a turn-off pulse having bi-polarity, supplied through the control loop, allows the capacitor $\mathrm{C} 1$ to be charged. However, for pre-charging, only a few turn-off pulses are sequentially applied to the gate driving circuit so as to charge only the voltage of the capacitor $\mathrm{C} 1$, while the semiconductor switch $\mathrm{Sw}$ is maintained in the turn-off state for safe operation. At this time, the flow of the current is as shown in Fig. 8 (c).

2) Turn-on pulse mode: When a turn-on pulse is applied to the gate drive circuit in a state where the capacitor $\mathrm{C} 1$ is fully charged, as shown in Fig. 8 (a), a pulse having a positive $(+)$ polarity is applied to the lower side of the control transformer to charge the capacitor $\mathrm{C} 1$ via the diode $\mathrm{D} 4$, and the zener diode D7 to be electrically conducted so as to turn on the switching transistor Q4. As the switching transistor Q4 is turned on, the voltage of the capacitor $\mathrm{C} 1$ is supplied to the GND via the resistor R4 and the diode D6. Thus, the voltage applied across the resistor R4 allows the switching transistor Q3 to be turned on. Similarly, the gate power charged in the capacitor $\mathrm{C} 1$ allows the current to flow through the GND via the switching transistor Q3 and the resistors R6 and R8. Finally, the voltage applied across the resistor R8 causes the semiconductor switch Sw to be turned on, and hence, the semiconductor switch $\mathrm{Sw}$ is maintained in the turn-on state. 


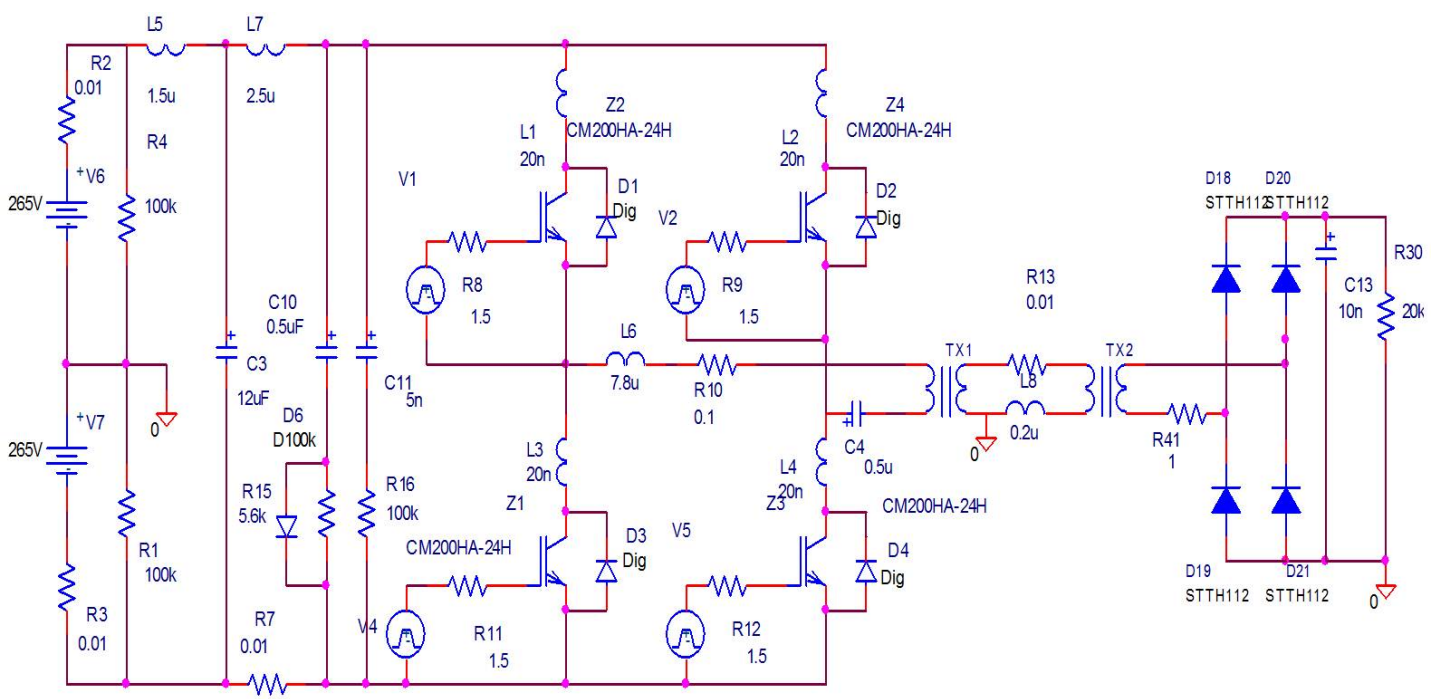

Fig. 3. PSpice simulation model for $15-\mathrm{kW}$ charger.

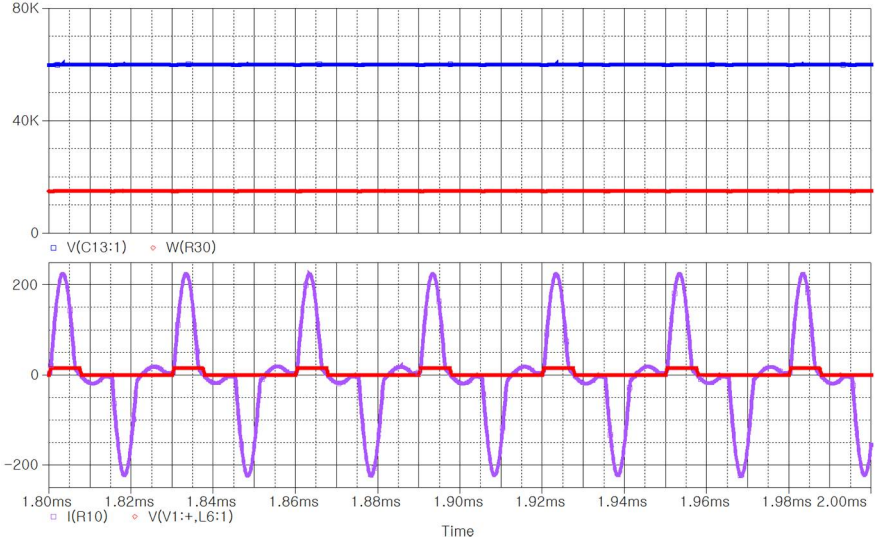

Fig. 4. Simulation results for resonant current.

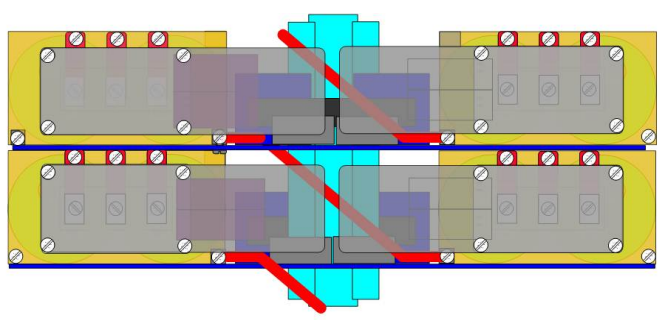

Fig. 5. Structure of power module.

3) Turn-on maintaining mode: Once the switching transistor Q3 is electrically conducted and the semiconductor switch $\mathrm{Sw}$ is turned on, the resistance between the drain and the source of the semiconductor switch $\mathrm{Sw}$ has a very small value because the IGBT switch is saturated. Although the turn-on pulse is extinguished, the gate power source allows a path to be formed to the GND via the resistors R4 and R5, the diode $\mathrm{D} 1$, and the semiconductor switch $\mathrm{Sw}$, as shown in Fig. 8 (b), so that the switching transistor Q3 continues to be electrically conducted. Therefore, the semiconductor switch $\mathrm{Sw}$ is maintained in the turn-on state until a turn-off pulse is applied to the gate driving circuit via the voltage applied across the resistor R8.

At this time, if an arc occurs at any load terminal to allow

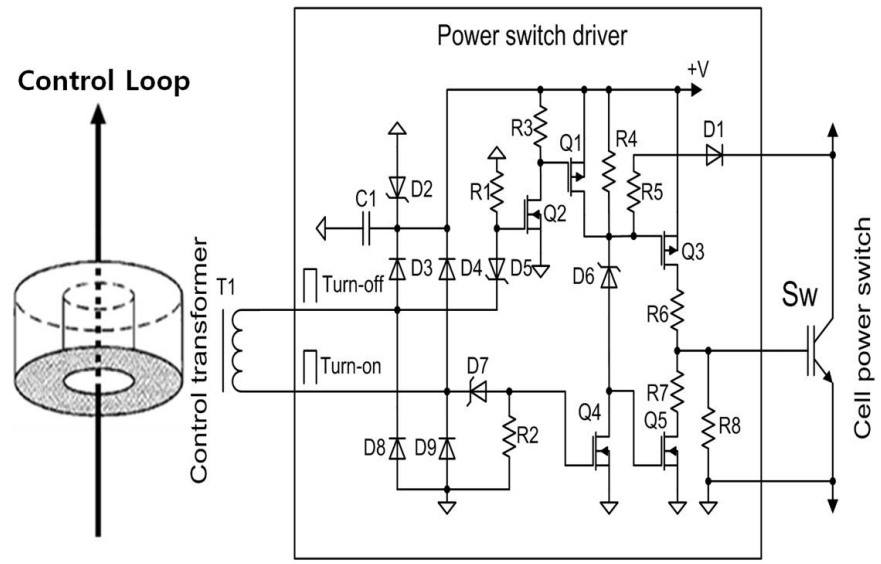

Fig. 6. Proposed gate driver circuit.

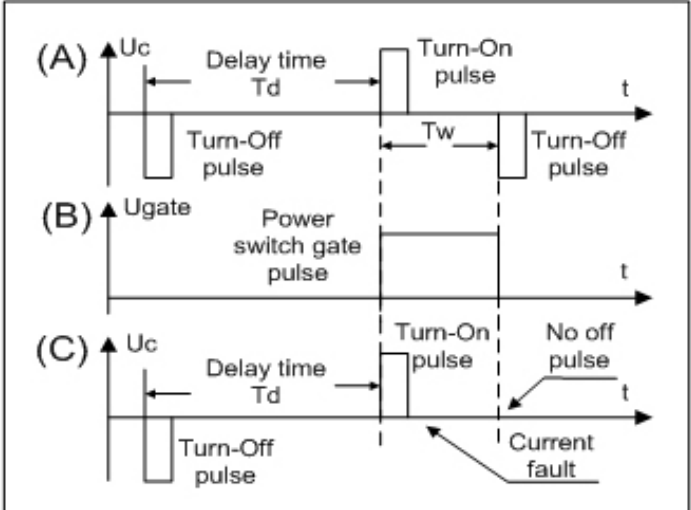

Fig. 7. Operational principle of driver

a short current to flow through the semiconductor switch Sw, although the internal resistance of the semiconductor switch $\mathrm{Sw}$ is small, the voltage drop across it will increase owing to the high short current causing the diode D1 to become reverse-biased and thereby turn off the switching transistor Q3. Consequently, the semiconductor switch $\mathrm{Sw}$ is turned off so as to protect the device from short circuits.

The semiconductor switch can be safely protected because 


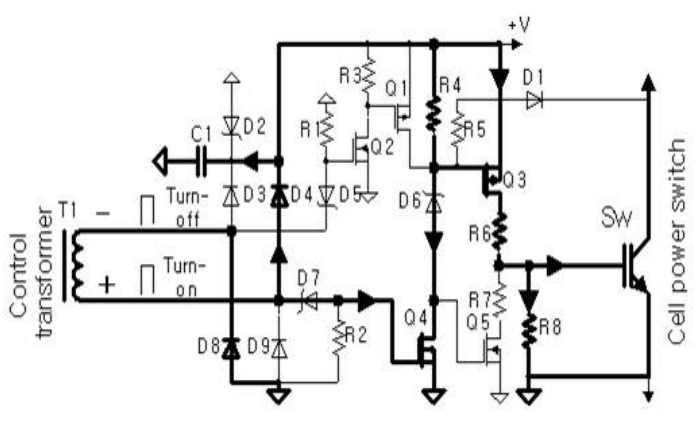

(a) Turn-on pulse mode.

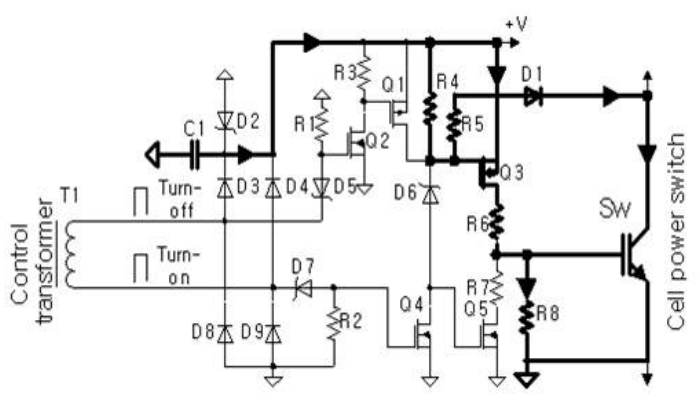

(b) Turn-on maintaining mode.

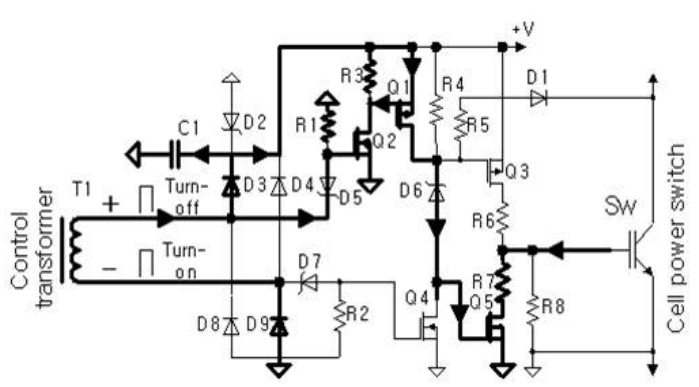

(c) Pre-charging \& turn-off mode.

Fig. 8. Operation modes of proposed gate driver circuit.

all of the transitions are performed smoothly.

4) Turn-off mode: When a turn-off pulse is applied to the gate driving circuit during the period in which the turn-on state is maintained, a pulse having a positive (+) polarity is applied to the upper end of the secondary winding of the control transformer to allow the capacitor $\mathrm{C} 1$ to be charged continuously, while allowing current to flow through the GND via the diode D5 and the resistor R1 to thereby turn on the switching transistor Q2. Therefore, the switching transistors Q1 and Q5 are sequentially turned on to cause the gate terminal of the semiconductor switch to be pulled down to the GND, so that the semiconductor switch and the switching transistor Q3 are sequentially turned off to cause the semiconductor switch to be maintained in the turn-off state until the next turn-on pulse is applied to the gate driving circuit. This is shown in Fig. 8 (c).

Hence, depending on the gate driving circuit, the control of the application time between the turn-on and turn-off pulses enables free adjustment of the pulse width.

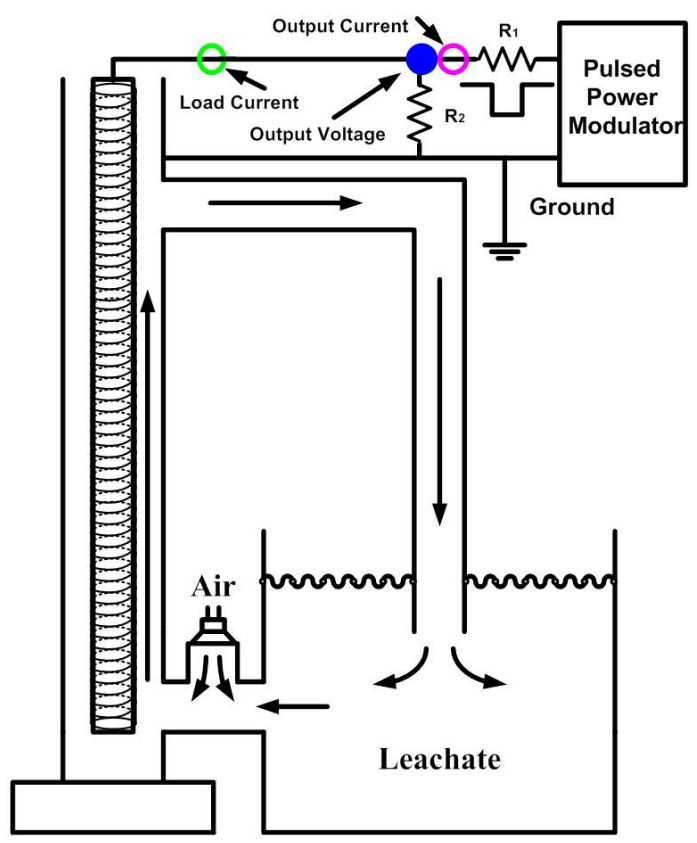

Fig. 9. Structure of proposed water treatment system.

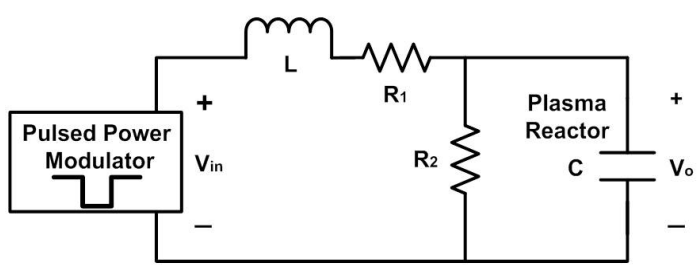

Fig. 10. Electrical equivalent circuit for pulsed-power system.

\section{Design And Analysis of the Water Treatment SYSTEM}

A sketch of the proposed water treatment system is shown in Fig. 9. The system consists of a leachate water storage tank, a circulation pump, an air inlet, a ballast resistor $\left(\mathrm{R}_{1}\right)$, a pull down resistor $\left(\mathrm{R}_{2}\right)$, and a pulsed-power modulator. To generate the desired output pulse on the plasma reactor side, it is necessary to determine appropriate values for the pull down and ballast resistors with respect to the line inductance and plasma reactor capacitance. Therefore, an electrical equivalent circuit model for the entire system is derived as shown in Fig. 10. $\mathrm{L}$ represents the total inductance from the modulator to the plasma reactor, including the inductance inside the modulator and the line inductance. The plasma reactor is replaced by a capacitor, which is constructed from two electrodes with airwater as the dielectric.

Based on the pulse state, which can be described by the rising, falling, and flat top regions, three types of equations were derived for the output voltage $\left(\mathrm{V}_{\mathrm{o}}\right)$ because the frequency components and level of the input voltage $\left(\mathrm{V}_{\text {in }}\right)$ are different for each region.

In the pulse-rising region, the Laplace domain output voltage equation with respect to the step input is calculated using the simple voltage divide rule from Fig. 4 and is expressed as:

$$
V_{o}(s)=\frac{V_{i n}}{s L C} \frac{1}{\left(s+\alpha_{1}\right)\left(s+\alpha_{2}\right)}
$$


TABLE III

SUMMARY OF ELECTRICAL EQUIVALENT CIRCUIT PARAMETERS FOR WATER TREATMENT SYSTEM

\begin{tabular}{|c|c|}
\hline Line inductance, $\mathrm{L}$ & $30 \mu \mathrm{H}$ \\
Plasma reactor capacitance, $\mathrm{C}$ & $700 \mathrm{pF}$ \\
Ballast resistance, $\mathrm{R}_{1}$ & $14 \Omega$ \\
Pull-down resistance, $\mathrm{R}_{2}$ & $1.5 \mathrm{k} \Omega$ \\
Resistive attenuation factor, $\mathrm{n}$ & 0.99 \\
Time constant, $\mathrm{T}$ & $0.9 \mu \mathrm{s}$ \\
Damping factor, $\mathrm{k}$ & 0.1 \\
Characteristic impedance, $\mathrm{Z}_{\mathrm{o}}$ & $207 \Omega$ \\
Induced voltage from modulator & $30 \mathrm{kV}$ \\
\hline
\end{tabular}

where, $\alpha_{1}, \alpha_{2}=\left(\frac{R_{1}}{2 L}+\frac{1}{2 R_{2} C}\right) \pm \sqrt{\left(\frac{R_{1}}{2 L}+\frac{1}{2 R_{2} C}\right)^{2}-\left(\frac{R_{1}+R_{2}}{R_{2} C L}\right)}$

It can be simplified by defining three constants, namely, the resistive attenuation factor $(\mathrm{n})$, the time constant $(\mathrm{T})$, and the damping factor $(\mathrm{k})$, which are closely related to the waveform of the output voltage.

$$
\alpha_{1}, \alpha_{2}=\frac{2 \pi k}{T} \pm j \frac{2 \pi}{T} \sqrt{(1-k)^{2}}
$$

where, $n=\frac{R_{2}}{R_{1}+R_{2}}, T=2 \pi \sqrt{n L C}, k=\frac{T}{4 \pi}\left(\frac{R_{1}}{L}+\frac{1}{R_{2} C}\right)$

Before solving the inverse Laplace transform of (1), it is necessary to assume that the value of the damping factor is less than 1 to provide the under damping response for the output voltage because it has a faster rising time when compared to the critical and over damping responses. This assumption imposes a constraint on the selection of the ballast and the damping resistor values. Finally, the time domain equation for the output pulse at the rising edge is derived as:

$$
\begin{aligned}
V_{o}(t) & =n V_{\text {in }}\left\{1-\left[\frac{k}{\sqrt{1-k^{2}}} \sin \left(\frac{2 \pi \sqrt{1-k^{2}}}{T}\right)\right.\right. \\
& \left.\left.+\cos \left(\frac{2 \pi t \sqrt{1-k^{2}}}{T}\right)\right] e^{-\frac{2 \pi k t}{T}}\right\}
\end{aligned}
$$

From (3), it is observed that the rising time of the induced pulse in the plasma reactor is proportional to the square root of $\mathrm{L}, \mathrm{C}$, and the resistive attenuation factor, $\mathrm{n}$. That is, a higher value of $R_{1}$ increases the rising time at a fixed $L, C$, and $R_{2}$. On the other hand, the efficiency of the overall system decreases owing to losses at $\mathrm{R}_{1}$. Moreover, considering the overshoot, which is closely related to the damping factor $\mathrm{k}$, a smaller value of $\mathrm{k}$ represents a larger overshoot. In order to understand the effect of each term, the definition of the damping factor can be expressed by $R_{1}, R_{2}$, and the characteristic impedance $\mathrm{Z}_{\mathrm{o}}$.

$$
k=\frac{\sqrt{n}}{2}\left(\frac{R_{1}}{Z_{o}}+\frac{Z_{o}}{R_{2}}\right)
$$

where, $Z_{o}=\sqrt{\frac{L}{C}}$

Finally, the time domain response for the induced voltage at the pulse rising edge was analyzed from the viewpoint of the rising time and overshoot with respect to the electrical circuit parameters.

The analysis of the flat-top region of the pulse is very simple because the high-frequency components are almost zero and only dc component exists in this region. This implies that the impedance of the reactive components can be ignored from the electrical equivalent circuit. Therefore, the applied voltage from the pulsed-power modulator is induced in the plasma

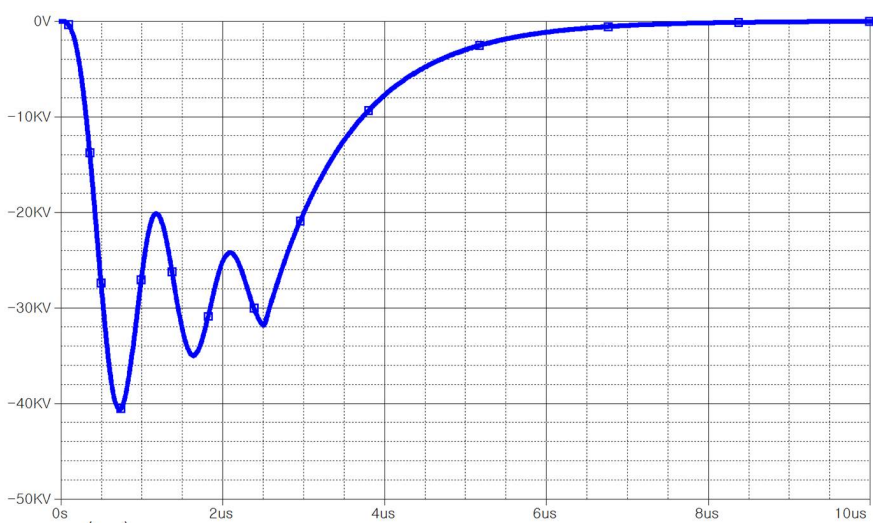

Fig. 11. PSpice simulation results for output voltage.

reactor only by the resistive divider and it is expressed as:

$$
V_{o}(t)=n V_{i n}(t)=\frac{R_{2}}{R_{1}+R_{2}} V_{\text {in }}(t)
$$

The falling edge response is important for increasing the pulse repetition rate because the energy stored in the plasma reactor should be discharged before the next pulse. In this region, the electrical circuit model can be simplified by using a simple $\mathrm{R}_{2}$ and $\mathrm{C}$ discharging circuit. Therefore, the tailing voltage duration is determined by the value of the pull-down resistor $R_{2}$. The simple expression for the falling edge is expressed as:

$$
V_{o}(t)=n V_{\text {in }}(t) e^{-\frac{t}{R_{2} C}}=\frac{R_{2}}{R_{1}+R_{2}} V_{\text {in }}(t) e^{-\frac{t}{R_{2} C}}
$$

From (6), it is clear that the value of $R_{2}$ should be determined by considering the maximum repetition rate of the induced voltage for a fixed plasma reactor capacitance $\mathrm{C}$.

Consequently, the value of each parameter is measured or determined from an analysis of the output voltage of the plasma reactor and it is summarized in Table III. In order to verify the derived equations and the determined values, a PSpice simulation model was developed. Fig. 11 shows the output voltage waveform from the PSpice simulation, and the measured rising time and peak value of output voltage are 500 $\mathrm{ns}$ and $40 \mathrm{kV}$, respectively, when a $30 \mathrm{kV}$ pulse is applied to the plasma reactor.

\section{EXPERIMENTAL RESULTS}

The experimental waveforms of the output pulses for the variable output voltage, the pulse width, and the frequency operation have been obtained previously [9]. Therefore, this paper only presents the waveforms of the modified parts, the charger, and the driver circuit. Fig. 12 shows the measured charging loop current, which represents the resonant current from the series resonant inverter. The frequency of the resonant current is similar to the designed value. Fig. 13 shows the control loop current and the output pulse voltage. Furthermore, the waveforms of the turn-on and turn-off control signals and the resultant gate voltages at the IGBT switch are shown in Fig. 14. This experimental waveform clearly indicates that the modified driver circuit functions satisfactorily from the viewpoint of the control loop signal and the power. A pulse voltage of $40 \mathrm{kV}, 2.5 \mu \mathrm{s}$ width, and $1 \mathrm{kHz}$ pulse repetition 


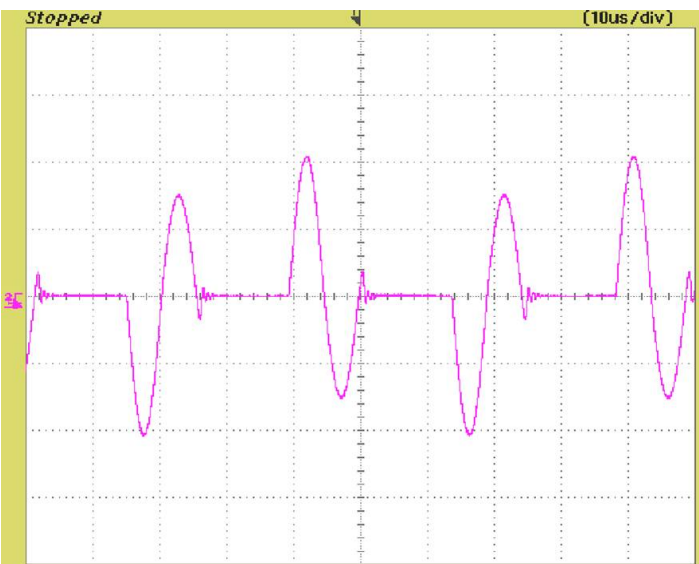

Fig. 12. Experimental waveform of charging loop current. (50 A/div., 10 $\mu \mathrm{s} /$ div.).

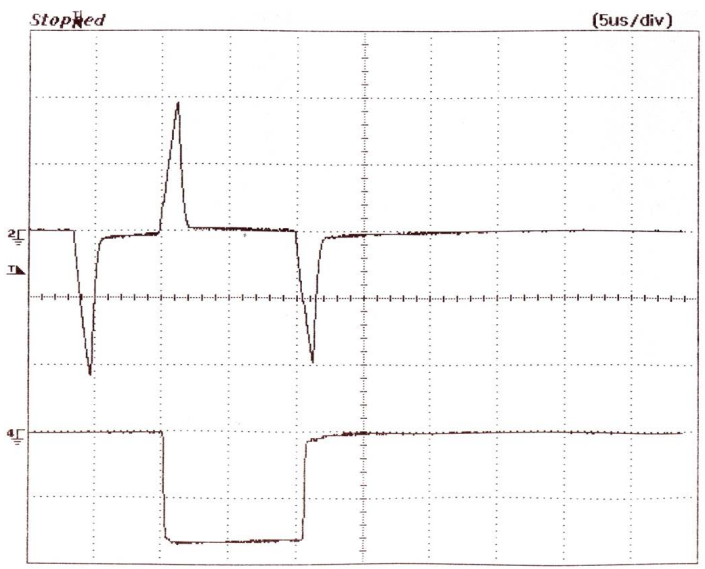

Fig. 13. Experimental waveform of control loop current and output pulse. (20 A/div., $10 \mathrm{kV} /$ div., $5 \mu$ s/div.).

rate was applied. As a result, the load current of the water treatment system reached up to $100 \mathrm{~A}$ with a rising time of 200 ns. This is shown in Fig. 15. Fig. 16 shows a comparison of photographs of the leachate water before and after treatment with a 10-min pulse at a 1-pps pulse repetition rate. The COD of the benzene series were found to be $150 \mathrm{ppm}$ before treatment and $8 \mathrm{ppm}$ after treatment, which satisfies the government regulation level of $15 \mathrm{ppm}$.

\section{CONCLusions}

In this paper, a pulsed-power-based leachate water treatment system has been proposed and tested. Leachate water treatment employing a high-voltage pulse is comparable to a conventional chemical reaction system from the viewpoint of total cost and effectiveness. The feasibility of the proposed scheme has been confirmed under laboratory conditions.

To realize an efficient pulsed-power water treatment system, an IGBT-based pulsed-power supply was introduced, including a high-efficiency charging inverter, an IGBT stacking structure, and a reliable gate driver circuit with a full-bridge inverter. The developed pulsed-power modulator can improve the system reliability via a unique arc protection algorithm.

The proposed pulsed-power water treatment system was analyzed under given conditions. Finally, the experimental results verify that the proposed system and analysis are feasible for water treatment.

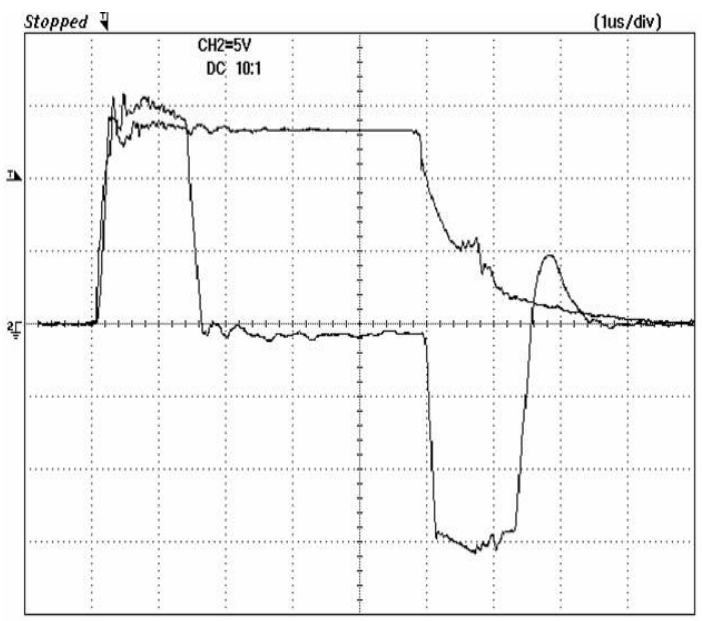

Fig. 14. Turn-on \& turn-off pulses and IGBT gate voltage. (5 V/div.).

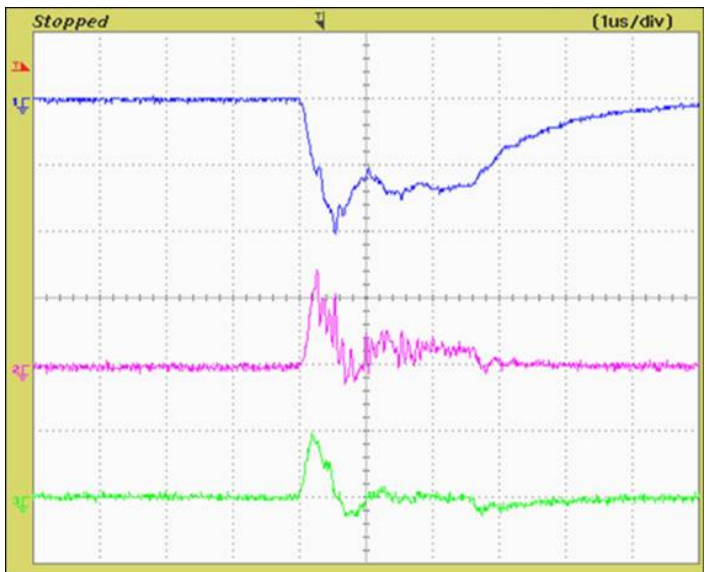

Fig. 15. Output waveforms of pulsed-power modulator for leachate water treatment. (1: output voltage ( $20 \mathrm{kV} /$ div. $), 2$ : output current (100 A/div.), 3: load current (100 A/div.)).

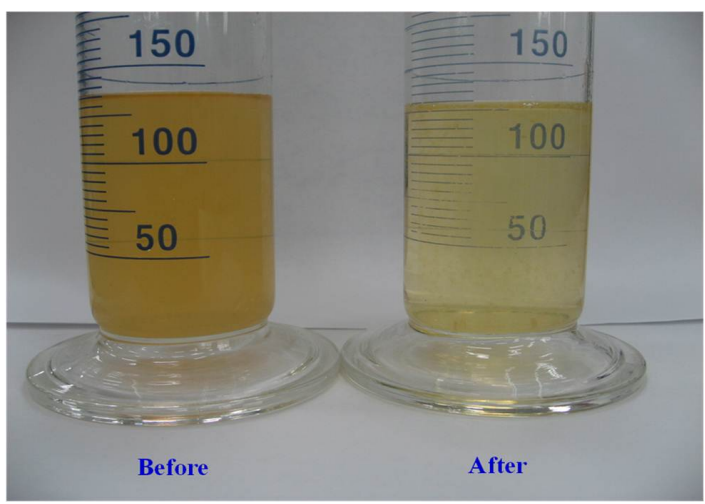

Fig. 16. Comparison of leachate water before and after treatment (COD of benzene series was detected to be $150 \mathrm{ppm}$ before treatment and $8 \mathrm{ppm}$ after treatment.). 
The development of positive-pulse-power treatment of water and a large plasma reactor design will be considered in future studies.

\section{REFERENCES}

[1] H. Akiyama, "Streamer discharges in liquids and their applications," IEEE Transactions on Dielectrics and Electrical Insulation, Vol. 7, No. 5, pp. 646-653, Oct. 2000.

[2] J. Kornev, "Electric discharge treatment of water containing organic substances," in Proceedings of the 7th Korea-Russia Symposium, pp. 243-247, 2003.

[3] C. Tamabe, S. Ihara, "Discharges in water and applications to wasted water treatment," IEEE T\&D Asia, 2009.

[4] T. Honda, Y. Minamitani, "The effect of a water-droplet spray and gas discharge in water treatment by pulsed power," IEEE Transactions on Plasma Science, Vol. 37, No. 1, pp. 179-183, Jan. 2009.

[5] G. H. Rim et al., "Semiconductor switch based pulsed power generator for plasma source ion implantation," IEEE International Power Modulator Conference, 2004

[6] G. H. Rim et al., "Solid state Marx generator using series connected IGBTs," IEEE International Power Modulator Conference, 2004.

[7] J. H. Kim, G. H. Rim et al., "IGBT stacks based pulsed power generator for PIII\&D," IEEE Pulsed Power Conference, 2005.

[8] H. J. Ryoo, G. Gussev, S. R. Jang, "Development of 10kV, 50A, 50kHz high repetitive pulsed power modulator based on IGBT stacks," IEEE International Power Modulators and High Voltage Conference, pp. 384387, 2008.

[9] H. J. Ryoo, G. Goussev, G. H. Rim, S.R. Jang, "Development of $60 \mathrm{kV}$, $300 \mathrm{~A}, 3 \mathrm{kHz}$ pulsed power modulator for wide applications," Proceedings of the 2th Euro-Asia Pulsed Power Conference, Vol. 115, No. 6, pp. 967970,2009

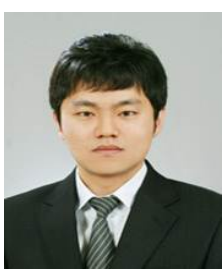

Sung-Roc Jang was born in Daegu, Korea, in 1983. He received his B.S. from Kyungpook National University, Daegu, Korea, in 2008, and his M.S. in Electronic Engineering from the University of Science \& Technology (UST), Deajeon, Korea, in 2010, where he is currently pursuing his Ph.D. His current research interests include high-voltage resonant converters and semiconductor-switch-based pulsed power modulators and their industrial applications. He is a member of the Korean Institute of Power Electronics (KIPE), and the Korean Institute of Electrical Engineers (KIEE).

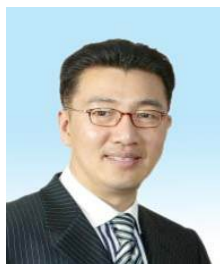

Hong-Je Ryoo received his B.S., M.S., and Ph.D. in Electrical Engineering from SungKyunkwan University, Seoul, Korea in 1991, 1995, and 2001, respectively. From 2004 to 2005, he was with WEMPEC at the University of Wisconsin-Madison, as a Visiting Scholar for his postdoctoral study. Since 1996, he has been with the Korea Electrotechnology Research Institute in Changwon, Korea. He is currently a Principal Research Engineer in the Industry Application Research Laboratory and a Leader of the Pulsed Power World Class Laboratory at that institute. Also, he has been an Associate Professor in the Department of Energy Conversion Technology, University of Science \& Technology, Deajeon, Korea, since 2005. His current research interests include pulsed power systems and their applications, as well as high power and high voltage conversions. Dr. Ryoo is a member of the Korean Institute of Power Electronics (KIPE), and the Korean Institute of Electrical Engineers (KIEE).

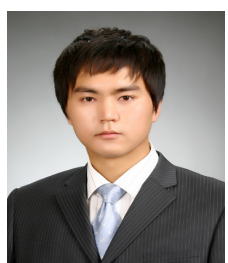

Seung-Bok Ok received his B.S. from Kyungpook National University, Daegu, Korea, in 2010, and he is currently pursuing his M.S. in Electronic Engineering at the University of Science \& Technology (UST), Deajeon, Korea. His current research interests include highvoltage resonant converters, solid-state pulsed power modulators and their applications. 